\title{
THE THANKSGIVING MEETING OF THE SOUTHWESTERN SECTION
}

The twenty-first regular meeting of the Southwestern Section was held at the University of Kansas on Saturday, December 1, 1928. The total attendance was forty-seven, including the following twenty-three members:

N. L. Anderson, Ashton, Wealthy Babcock, Florence Black, Brenke, Gaba, Garrett, Harshbarger, Hyde, Louis Ingold, Kempner, Luby, McShane, U. G. Mitchell, Mossman, O. J. Peterson, Richert, Roever, G. W. Smith, Stouffer, J. S. Turner, J. J. Wheeler, Wyant.

Professor Mitchell occupied the chair, being relieved during the morning session by Professor Roever. The morning session was devoted to the reading of the papers listed below. During the afternoon session Professor A. J. Kempner gave a special lecture by invitation of the program committee on The development of the analytical theory of numbers in the present century.

The titles and abstracts of the papers read are given below. The papers of Altshiller-Court, Reid, and Whyburn were read by title.

1. Professor Louis Ingold: Generalization of the tensor laws of transformation.

In the theory of tensors two types of transformation of sets of quantities are employed, namely, the covariant, and the contravariant laws of transformation. These involve the first derivatives of the new variables with respect to the old, or of the old with respect to the new. In connection with certain invariant expressions there occur other types of transformation involving derivatives of one set of variables with respect to the other, of order higher than the first. These are the generalizations considered in this paper.

2. Miss Nola L. Anderson: First normal spaces in Riemannian geometry.

If a space is defined by a general vector function of the coordinates $u^{i}$, say $f\left(u^{1}, u^{2}, \cdots, u^{n}\right)$, the first derivatives $f_{i}=\partial f / \partial u^{i}$ of $f$ at each point determine the tangent space. Every vector orthogonal to the tangent space is called a normal vector. A normal vector expressible in terms of the tangent vectors $f_{i}$ and their derivatives is called a first normal. It is the pur- 
pose of this paper to investigate the relations connecting various first normal vectors invariant under change of coordinates. Some of these normals are independent of any loci passing through the point under consideration while others are associated with curves, surfaces or other loci passing through the point. The details are given only for two- and three-dimensional spaces.

3. Professor U. G. Mitchell: Note on Mersenne numbers.

At the February 1925 meeting of the Kansas Section of the Mathematical Association of America the author presented a paper summarizing the results previously obtained and the chief methods employed in factoring Mersenne and Fermat numbers. A simple method of attack based upon geometrical considerations was suggested and some results obtained by the method were also presented. In the present note is shown as a further result obtained by the method the fact that $2^{1321}-1$ has the factor 7927 , proof of which is established by two independent series of congruences.

4. Professor W. C. Brenke: On polynomial solutions of a class of linear differential equations of the second order.

In this paper certain polynomial solutions of the differential equation $p y^{\prime \prime}+q y^{\prime}+r_{n} y=0$ are considered, when $p$ and $q$ are polynomials in $x$ and $r_{n}$ a function of $n$ alone. In particular, simple formulas for the normalizing constants and generating functions are obtained, the latter by an application of the Laplace expansion as used by Darboux for the Jacobi polynomials.

\section{Professor Nathan Altshiller-Court: Some tetrahedral} complexes.

A tetrahedral complex is defined as the totality of straight lines which cut the four faces of a tetrahedron in four points having a constant anharmonic ratio. The author proves several theorems on such complexes.

6. Professor W. H. Roever: A geometric representation of a line integral.

By regarding the differential expression $P d x+Q d y$ as defining surface elements in space which cut from a vertical cylindrical surface of directrix $c$ in the $x y$ plane lineal elements which have for unions a one-parameter family of curves on this cylindrical surface, it is possible to give a very simple geometrical representation to the value of the line integral $\int_{c} P d x+Q d y$ and to see geometrically under what conditions this line integral is zero for a closed path.

7. Professor Wealthy Babcock: On the geometry associated with certain determinants with linear elements.

If the linear elements of the determinantal form in three variables are set equal to zero, each represents a straight line. The geometry of these lines may be studied by placing restrictions on the invariants and covariants of the linear forms which are the elements of the determinant. Certain 
facts concerning the geometry of the lines which are obtained from the elements of the determinantal form of the second order and their relation to the conic, and of the lines which are obtained from the determinantal form of the third order and their relation to the plane cubic curve were brought out in this paper. From the determinantal form of the equations of plane curves, some theorems concerning the generation of plane curves of order $n$ by projective pencils of curves of order less than $n$, may be readily proved.

8. Professor J. S. Turner: The factoring of large numbers by means of positive determinants of Seelhoff type.

This is a continuation of former papers (this Bulletin, vol. 37, pp. 163, 399). A list (probably complete) of 14 positive determinants $D$ is given, for which: (a) $D$ contains no square factor, $(b) T^{2}-D=1,(c)$ there are two cycles of reduced forms in each genus. The method of applying these determinants to the factorization of large numbers is explained. The prime or composite character of a number of 10 digits can thus be determined in two or three hours.

9. Professor J. S. Turner: The expression of numbers from 12,000 to 30,000 in the form $w^{3}+x^{3}+2 y^{3}+2 z^{3}$.

The computation was undertaken at the instance of Professor L. E. Dickson as part of a project to verify von Sterneck's table (Wiener Sitzungsberichte, vol. 112, II $a$, (1903), pp. 1627-66) showing the number of cubes required to represent all numbers $\leqq 40,000$. The method adopted is described in the American Mathematical Monthly (vol. 35 (1928), p. 113). The verification, now completed, that every number following 8041 up to 40,000 is the sum of six cubes is of the greatest importance, since this result is employed in the proof of Waring's theorem that every number is the sum of nine cubes. In addition to $p=(111111111)$ the following theorems (L. E. Dickson, American Mathematical Monthly, April, 1927, p.177) are rendered independent of von Sterneck's table: $p=(11111112)$ (that is, Theorem III), $p=(1111114)$, where $p$ is any positive integer $\leqq 40,000$. These theorems are likewise true of the forms derived from them by partitioning 2 and 4 .

10. Professor E. B. Stouffer: A canonical expansion associated with developable surfaces.

The author obtains a canonical expansion for the equation of a developable surface by means of the fourth order differential equation associated with the edge of regression and also determines the geometrical location of the vertices of the associated tetrahedron of reference. All the work is far more simple than when the developable surface is treated as a special case of a curved surface, the method which has been used previously.

11. Mr. W. T. Reid: Countable differential systems containing a parameter.

A system of integral equations of the form $y(x, \mu)=\int_{0}^{x} A(t, \mu) y(t, \mu) d t$ 
$+a(\mu)$ is considered, where $A(x, \mu) \equiv\left(A_{i j}(x, \mu)\right)$ is a square matrix with a countable number of rows and columns, $y(x, \mu)=\left(y_{i}(x, \mu)\right)$ and $a(\mu) \equiv\left(a_{i}(\mu)\right)$, $(i, j=1,2, \cdots)$. We will call a solution of this system a set of functions $y_{i}(x, \mu), i=1,2, \cdots$, each of which is absolutely continuous in $x$ for fixed value of $\mu$ and such that $y(x, \mu)$ is a vector in Hilbert space. If (1) for each $\mu$ on $L, L_{1}<\mu<L_{2}, A_{i j}(x, \mu)$ is a measurable function on $X, 0 \leqq x \leqq 1$; (2) $a(\mu)$ for $\mu$ on $L$ is a single valued vector in Hilbert space; (3) there exists a non-negative summable function $\phi(x)$ on $X$ such that for each vector $\omega$ in Hilbert space $|A(x, \mu) \omega| \leqq \phi(x)|\omega|$; then there exists a unique solution of this system on $X L$. Sufficient conditions are given for the solution $y(x, \mu)$ to be continuous in $(x, \mu)$ on $X L$, and also to possess a partial derivative with respect to the parameter $\mu$. The above system is also considered for each element of the matrix $A(x, \mu)$ analytic in the parameter $\mu$.

12. Mr. W. T. Reid: Matrix solutions of countable differential systems and their adjoints.

If $A(x)$ is a square matrix in a countable number of dimensions limited by a summable function $\phi(x)$ on $X: a \leqq x \leqq b$, that is, for every pair of points $\xi$ and $\eta$, of Hilbert space and for every $n,\left|\sum_{i j=1}^{n} A_{i j}(x) \xi_{i} \eta_{j}\right| \leqq \phi(x)|\xi||\eta|$, then there exists a matrix $Y(x)$ of absolutely continuous functions satisfying equation (1): $Y^{\prime}(x)=A(x) \cdot Y(x)$ "almost everywhere" on $X$. It is proved that if $Y(x)$ is limited at some point of $X$, then $Y(x)$ is limited uniformly on $X$. Similarly, there exists an absolutely continuous matrix $Z(x)$ satisfying the adjoint equation (2): $Z^{\prime}(x)=-Z(x) \cdot A(x)$ "almost everywhere" on $X$, and if $Z(x)$ is limited at a point of $X$, then it is limited uniformly on $X$. If $Y(x)$ is limited and at a point of $X$ possesses a unique limited reciprocal, then $Y(x)$ possesses on $X$ a unique, limited, absolutely continuous reciprocal which is a solution of (2). We will call a matrix solution of (1) a limited, absolutely continuous matrix which satisfies (1) "almost everywhere" on $X$ and has a unique reciprocal. If $Y(x)$ is a matrix solution of (1) the most general matrix solution is $Y(x) \cdot C$ where $C$ is a constant limited matrix possessing a unique reciprocal. Furthermore, if $Z(x)$ is a matrix and $Z(x) \cdot Y(x)=C$ on $X$, where $Y(x)$ is a matrix solution on (1) and $C$ is a constant matrix possessing a unique reciprocal, then $Z(x)$ is a matrix solution of (2).

13. Professor G. T. Whyburn: On regular points of continua and regular curves of at most order $n$.

This paper appears in full in the present issue of this Bulletin.

E. B. Stouffer, Secretary of the Section 